\title{
Contribution of a Novel B3GLCT Variant to Peters Plus Syndrome Discovered by a Combination of Next-Generation Sequencing and Automated Text Mining
}

\author{
Justyna Totoń-Żurańska ${ }^{1}$, Przemysław Kapusta ${ }^{1}$, Magda Rybak-Krzyszkowska ${ }^{2}$, \\ Katarzyna Lorenc ${ }^{3}$, Julita Machlowska ${ }^{1}$, Anna Skalniak ${ }^{4}$, Erita Filipek ${ }^{5}$ (D) Dorota Pawlik $^{3}$ \\ and Paweł P. Wołkow ${ }^{1, *(D)}$ \\ 1 Center for Medical Genomics-OMICRON, Jagiellonian University Medical College, ul. Kopernika 7c, \\ 31-034 Krakow, Poland; jzuranska@gmail.com (J.T.-Ż.); przemyslaw.kapusta@uj.edu.pl (P.K.); \\ julita.machlowska@gmail.com (J.M.) \\ 2 Department of Obstetrics and Perinatology, The University Hospital in Krakow, ul. Kopernika 23, \\ 31-501 Krakow, Poland; rybaczka@interia.pl \\ 3 Department of Neonatology, Jagiellonian University Medical College, ul. Kopernika 23, \\ 31-501 Krakow, Poland; lorenc.kasia@poczta.onet.pl (K.L.); dorota.pawlik@uj.edu.pl (D.P.) \\ 4 Department of Endocrinology, Jagiellonian University Medical College, ul. Kopernika 17, \\ 31-501 Krakow, Poland; anna.skalniak@uj.edu.pl \\ 5 Clinic and Department of Pediatric Ophthalmology, School of Medicine in Katowice, \\ Medical University of Silesia in Katowice, ul. Ceglana 35, 40-514 Katowice, Poland; erita.filipek@gmail.com \\ * Correspondence: pawel.wolkow@uj.edu.pl
}

Received: 10 September 2019; Accepted: 26 November 2019; Published: 28 November 2019

\begin{abstract}
Anterior segment dysgenesis (ASD) encompasses a spectrum of ocular disorders affecting the structures of the anterior eye chamber. Mutations in several genes, involved in eye development, are implicated in this disorder. ASD is often accompanied by diverse multisystemic symptoms and another genetic cause, such as variants in genes encoding collagen type IV. Thus, a wide spectrum of phenotypes and underlying genetic diversity make fast and proper diagnosis challenging. Here, we used AMELIE, an automatic text mining tool that enriches data with the most up-to-date information from literature, and wANNOVAR, which is based on well-documented databases and incorporates variant filtering strategy to identify genetic variants responsible for severely-manifested ASD in a newborn child. This strategy, applied to trio sequencing data in compliance with ACMG 2015 guidelines, helped us find two compound heterozygous variants of the B3GLCT gene, of which c. $660+1 \mathrm{G}>\mathrm{A}$ (rs80338851) was previously associated with the phenotype of Peters plus syndrome (PPS), while the second, NM_194318.3:c.755delC (p.T252fs), in exon 9 of the same gene was noted for the first time. PPS, a very rare subtype of ASD, is a glycosylation disorder, where the dysfunctional B3GLCT gene product, O-fucose-specific $\beta$-1,3-glucosyltransferase, is ineffective in providing a noncanonical quality control system for proper protein folding in cells. Our study expands the mutation spectrum of the B3GLCT gene related to PPS. We suggest that the implementation of automatic text mining tools in combination with careful variant filtering could help translate sequencing results into diagnosis, thus, considerably accelerating the diagnostic process and, thereby, improving patient management.
\end{abstract}

Keywords: clinical genetics; diagnosis; anterior segment disease; ophthalmology 


\section{Introduction}

In recent years, next-generation sequencing technology (NGS) and access to databases containing sequencing data from patients with eye disorders have accelerated research in ophthalmic molecular genetics. However, still the most difficult and time-consuming part of the work is data analysis and clinical interpretation of reported variants, especially in cases with blended phenotype, when the decision on major clinical feature, crucial for diagnostic process is difficult to make. Additionally, due to massive sequencing data generation and relatively restricted curation, available databases, within reliable information contain false variant pathogenicity attributions. Thus, rigorous variant interpretation, as recommended by the American College of Medical Genetics and Genomics (ACMG) [1], followed by extensive review of the existing literature could improve diagnostic accuracy.

Anterior segment dysgenesis (ASD) encompasses a spectrum of ocular disorders affecting the structures of the anterior eye chamber, including the iris, lens, cornea, and the anterior chamber angle. Clinical manifestations include corneal opacities, cataracts, and iridocorneal adhesions. ASD poses a high risk of the patient developing early onset and aggressive glaucoma resulting from dysregulation of aqueous humor flow, high intraocular pressure (IOP), and death of retinal ganglion cells [2]. Disorders related to such a phenotype include Axenfeld Rieger syndrome (ARS), isolated Peters Anomaly, Peters plus syndrome (PPS), primary congenital glaucoma, congenital hereditary endothelial dystrophy, and iridogoniodysgenesis anomaly [3], all of which are registered as rare by Orphanet. The genetic background of ASD is only partially known and may be related to the disruption of many genes, e.g., CYP1B1, BMP4, FOXC1, PAX6, FOXE3, NDP, SLC4A11, HCCS, PITX2, PITX3, LMX1B, and PXDN [4-8], several of which can modulate tyrosinase activity, postulated as an important modulator of iridocorneal angle malformations [6]. Importantly, diseases with multisystemic manifestations can also present with ASD, e.g., syndromes resulting from mutations in genes encoding collagen type IV: COL4A1 and COL4A2 [2,9,10], often manifest with neurological disorders, what may hinder finding the relevant genetic cause of the disease. Diseases from the ASD spectrum may be dominant (ARS, aniridia) or recessive (primary congenital glaucoma, PPS), and due to the diverse genetic background, several phenotypes may present with either mode of inheritance [11].

Due to the heterogeneous clinical manifestation of the syndrome, reflecting the complex genetic background, classification, and proper diagnosis of ASD is still challenging. Here, we report a strategy for NGS data analysis in order to find the genetic cause of a rare combination of symptoms in a pediatric patient with a set of ocular disorders, cleft lip and high-arched palate, using data from DNA sequencing of coding regions of 4813 genes in both parents and the affected child.

We demonstrate a variant filtering strategy combined with information on the observed phenotype coded in Human Phenotype Ontology (HPO) terms and information enrichment by literature mining with the AMELIE (Automatic MEndelian LIterature Evaluation) [12] tool and by genomic variant annotation and prioritization with wANNOVAR [13-15] to help draw biological insights from sequencing data and increase the chances of faster and accurate diagnosis. Literature mining tool prioritizes variants according to the current knowledge from published data and enables critical verification of results through linking ranked genes important for the phenotype with relevant publication, what accelerates the process and may reduce false positive findings. In our opinion, this analytical strategy should be applicable to other clinical scenarios with complex genetic background and heterogeneous clinical manifestations also.

\section{Results}

\subsection{Case History and Clinical Findings}

\subsubsection{Obstetric Information}

The 30-year-old pregnant patient was referred for the first-trimester testing due to three previous miscarriages and advanced maternal age. The parents had one healthy child. Screening involved testing 
for trisomies 21,18 , and 13, ultrasonography, measurement of maternal serum free $\beta$-human chorionic gonadotropin ( $\beta$-hCG), and pregnancy-associated plasma protein-A (PAPP-A) levels, according to the Fetal Medicine Foundation recommendations. Both biochemical parameters were measured on the DELFIA Xpress analyzer (Perkin Elmer). The mother denied medication history or infection during pregnancy. Moreover, no familial predisposition to any disease was identified. Routine fetal scanning at 13 weeks of gestation showed a cleft lip and palate and increased nuchal translucency (Figure 1). Nuchal translucency was assessed as $3 \mathrm{~mm}$ and was estimated as being over the 95th percentile for the given crown-rump length $(C R L=67.7 \mathrm{~mm})$. No other anomalies were detected for the fetus. Free $\beta$-hCG was $1.472 \mathrm{MoM}$ (Multiple of Median) and PAPP-A, $0.634 \mathrm{MoM}$. The first trimester combined with prenatal screening yielded a risk of 1:149 for trisomy 21, 1:7780 for trisomy 18, and 1:7447 for trisomy 13.

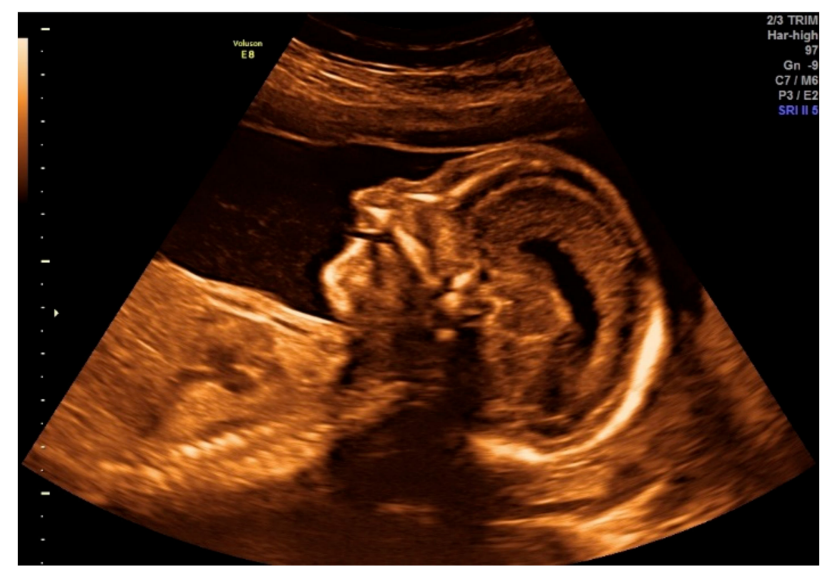

Figure 1. Obstetric sonogram of the fetus at 13 weeks of gestation.

Having detected a fetal defect and correlated it with an indirect risk group, an invasive diagnostic procedure was suggested to determine the fetal karyotype. Amniotic fluid was aspirated during amniocentesis on week 15, day 4 of pregnancy. Following amniocyte culture, a karyotype of a normal male $(46, \mathrm{XY})$ was obtained at a resolution of 550 bands. The couple was then referred for genetic counseling for further etiological investigation.

The second ultrasonographic examination performed at the 20th week of gestation, as per the recommendations of the Polish Society of Gynecologists and Obstetricians, confirmed the presence of bilateral cleft lip and palate.

The patient (mother) was hospitalized with intrauterine growth restriction in the 28th week of gestation. In view of abnormal flow in the umbilical artery and the middle cerebral artery, as well as deteriorating fetal well-being, it was decided to terminate the pregnancy and perform a cesarean section.

\subsubsection{Initial Postnatal Examination}

Physical examination of the fetus revealed bilateral cleft alveolar processes, cleft lip, and high-arched palate. Rhizomelic proximal extremities and brachydactyly were noted. Microphthalmia and bilateral corneal opacities were observed during ophthalmic examination.

\subsubsection{Ophthalmic Evaluation}

Visual acuity in both eyes was determined as dubious light perception. Examination under general anesthesia resulted in the following findings (Figure 2). 

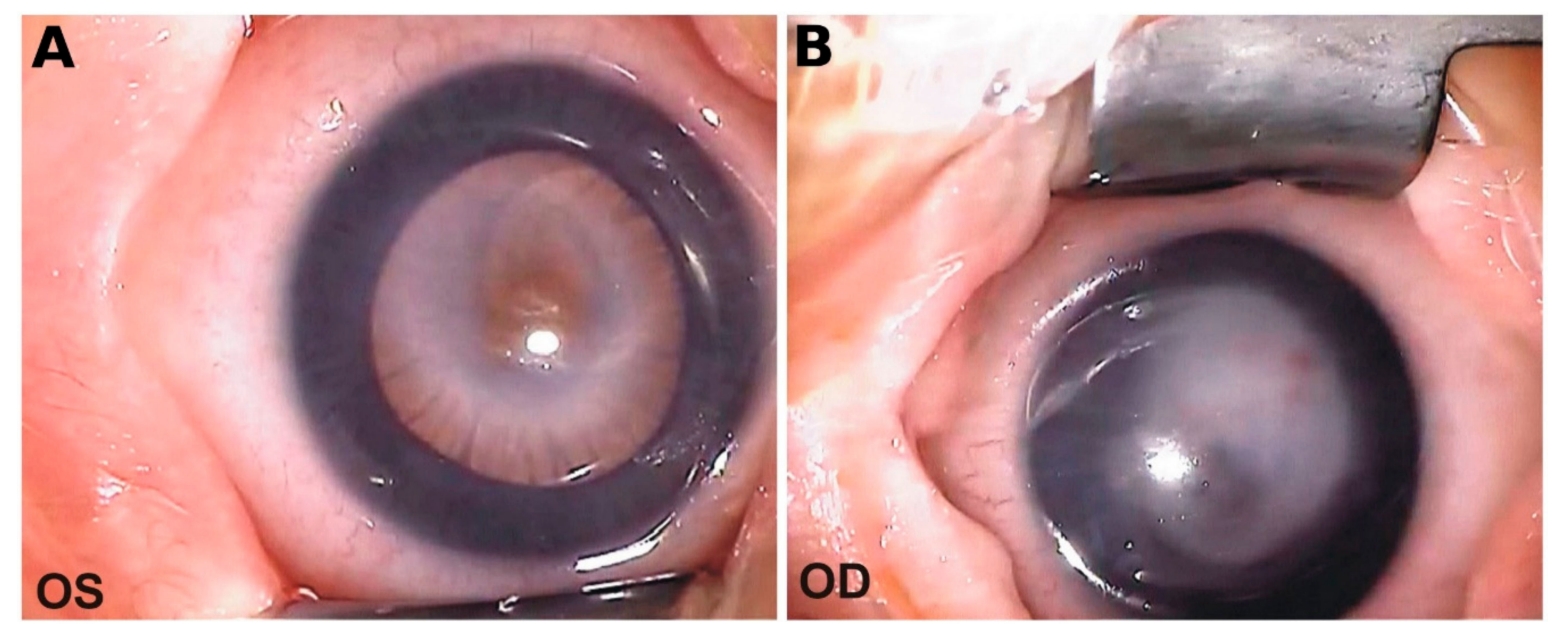

Figure 2. Anterior segment images of left (A) and right (B) eyes. Scale bars represent $2 \mathrm{~mm}$.

Right eye (RE): Cornea dimensions 8 x $8.5 \mathrm{~mm}$; IOP $10 \mathrm{mmHg}$; central corneal leucoma, subsequent to ulceration with ingrowth of blood vessels. Translucent peripheral cornea revealed flattened anterior eye chamber, medium-width iris with persistent pupillary membrane, and lens adhesion to the posterior corneal surface. A visible fragment of the lens was translucent with pink reflection from the eye fundus. Further details were hard to evaluate.

Left eye (LE): Cornea dimensions $8 \times 8.5 \mathrm{~mm}$; IOP $12 \mathrm{mmHg}$, partial central leucoma. The peripheral part of the cornea was translucent with a persistent pupillary membrane. The pupillary dilatory response was normal and some peripheral blood vessels of the retina could be visualized.

The anatomical axial length of the eyeballs in ultrasonographic (USG) evaluation was $18 \mathrm{~mm}$ (RE) and $17.5 \mathrm{~mm}$ (LE) in projection AB (Ultrascan B, Alcon, USA). Diagnostic USG of the posterior part of the eyes revealed no pathology in RE and a small floater above an optic nerve disk in LE.

The patient remained under ophthalmic supervision. At the age of four months, central thinning of the corneal leucoma of the RE, threatening perforation and secondary glaucoma were determined in the RE. Corneal thickness in the RE was $826 \mu \mathrm{m}$ in the periphery but only $158-380 \mu \mathrm{m}$ in the center while being $855 \mu \mathrm{m}$ in the entire LE. IOP was $37 \mathrm{mmHg}$ in the right and $17 \mathrm{mmHg}$ in the LE. Amniotic membrane transplantation to protect the cornea and cyclocryotherapy to lower IOP were performed in the RE. Topical IOP lowering medications were started and the patient was followed for local status and IOP. In spite of ongoing pharmacological treatment, another round of amniotic membrane transplantation and cyclocryotherapy had to be performed at the age of six months. Stabilization of the IOP was achieved at the level of $26 \mathrm{mmHg}$ in the right and $18 \mathrm{mmHg}$ in the LE. The patient remains under ophthalmic supervision with permanent pharmacological medications to prevent glaucoma of the RE.

\subsection{High-Throughput Sequencing Results}

On average, 9.8 million reads were sequenced per sample, and approximately 8.9 million reads (91.1\%) were mapped on the reference using our custom pipeline. A high depth of coverage (at least $20 \times$ ) was maintained for $86.9 \%$ of the targeted region and approximately $95 \%$ of the target region was covered at least $10 \times$. In total, we detected 32,222 variants: 26,230 single nucleotide polymorphisms (SNPs), 2536 insertions, and 3456 deletions.

Following the GEMINI trio analysis, we identified 265 variants in total, of which 36 were de novo variants, 200 autosomal recessive variants, and 16 pairs of compound heterozygotes (32 variants, of which three were de novo) in the proband. In terms of localization, 38 variants were annotated as exonic and 227 as intronic changes. All discovered variants were concatenated into one VCF file for further analysis (Supplement 1). Using in silico prediction algorithms such as SIFT, PROVEAN, FATHMM-XF, and MutationTaster, we were able to select several candidates with possible damaging impact (Table 1). 
For genomic variant prioritization, we uploaded the generated VCF file (Supplement 1) into AMELIE and wANNOVAR with the following HPO identifiers: Corneal opacity (HP:0007957), abnormality of the anterior chamber (HP:0000593), anterior chamber synechiae (HP:0007833), and cleft lip/palate (HP:0000202). Based on AMELIE and wANNOVAR results (Table 2), we discovered that variants in both alleles of B3GLCT (alias B3GALTL) that were segregated from an unaffected father and unaffected mother, were strong candidates to be potentially responsible for the observed pathogenicity, which, most likely, was PPS (Supplement 2).

The first variant, inherited from the mother, was a substitution, NM_194318.3:c.660+1G>A (rs80338851), reported as pathogenic allele (B3GALTL, OMIM:610308:0001, RCV000001326.4) associated with PPS. Moreover, using Varsome, the discovered variant was classified according to ACMG2015 as pathogenic, with identified criteria to be PVS1, PP3, PP5.

The second variant, inherited from the father, was a deletion, NM_194318.3:c.755delC (p.T252fs), which has not been previously reported (according to LOVD, and is not present in the gnomAD database). In Varsome, the discovered variant was classified according to ACMG2015 as likely pathogenic, with identified criteria to be PVS1 and PM2. Moreover, this mutation had a MutationTaster score of 1 (prediction: Disease causing) and changed the reading frame downstream of the amino acid (AA) at position 252. As a result, the mutation terminated translation at AA position 264, whereas a wild-type protein is $498 \mathrm{AA}$ long. Both mutations were manually reviewed in an integrative genomic viewer (IGV). 
Table 1. Mutations discovered in the proband with possible damaging impact according to in silico analysis. Variants were sorted according to genomic coordinates.

\begin{tabular}{|c|c|c|c|c|c|c|c|c|c|c|}
\hline Gene & Mutation & $\begin{array}{l}\text { Amino Acid } \\
\text { Change }\end{array}$ & Zygosity & $\begin{array}{l}\text { Segregation } \\
\text { Analysis }\end{array}$ & $\begin{array}{c}\text { gnomAD } \\
\text { European MAF }\end{array}$ & SIFT & PROVEAN & FATHMM-XF & $\begin{array}{c}\text { Mutation } \\
\text { Taster }\end{array}$ & dbSNP \\
\hline \multirow{2}{*}{ LRPB } & NM_004631:c.73_74delCA & p.Gln $25 \mathrm{fs}^{*} 10$ & \multirow{2}{*}{$\begin{array}{l}\text { Compound } \\
\text { Heterozygous }\end{array}$} & De novo & $3.55 \times 10^{-4}$ & - & - & - & $\begin{array}{l}\text { Disease } \\
\text { causing }\end{array}$ & rs1491461533 \\
\hline & NM_004631:c.71delT & p.Leu $24 \mathrm{fs}^{*} 50$ & & De novo & $1.79 \times 10^{-4}$ & - & - & - & $\begin{array}{l}\text { Disease } \\
\text { causing }\end{array}$ & rs761955852 \\
\hline \multirow[b]{2}{*}{ TTN } & NM_133437:c.71141G>A & p.Arg23714His & \multirow[b]{2}{*}{$\begin{array}{l}\text { Compound } \\
\text { Heterozygous }\end{array}$} & Maternal & $2.88 \times 10^{-3}$ & Tolerated & Neutral & Benign & Polymorphism & rs200650668 \\
\hline & NM_133437:c.25190G>T & p.Ser8397Ile & & Paternal & $8.52 \times 10^{-4}$ & - & Deleterious & - & $\begin{array}{l}\text { Disease } \\
\text { causing }\end{array}$ & rs200335120 \\
\hline DMXL1 & NM_005509:c.7403_7405delATG & p.Asp2468del & Heterozygous & De novo & $9.30 \times 10^{-5}$ & - & Deleterious & - & $\begin{array}{l}\text { Disease } \\
\text { causing }\end{array}$ & rs200335120 \\
\hline \multirow{2}{*}{ LAMA4 } & NM_001105206:c.4665+7T>C & - & \multirow{2}{*}{$\begin{array}{l}\text { Compound } \\
\text { Heterozygous }\end{array}$} & Maternal & $1.80 \times 10^{-5}$ & - & - & Pathogenic & $\begin{array}{l}\text { Disease } \\
\text { causing }\end{array}$ & rs751477013 \\
\hline & NM_001105206:c.3239G>A & p.Arg1080Gln & & Paternal & $1.39 \times 10^{-2}$ & Damaging & Neutral & Pathogenic & $\begin{array}{l}\text { Disease } \\
\text { causing }\end{array}$ & rs41289902 \\
\hline$D E A F 1$ & NM_021008.3:c.69_98del30 & p.Ala24_Ala33del & Heterozygous & De novo & $1.06 \times 10^{-3}$ & - & - & - & $\begin{array}{l}\text { Disease } \\
\text { causing }\end{array}$ & rs766934551 \\
\hline GYS2 & NM_021957:c.421G>A & p.Gly141Ser & Homozygous & $\begin{array}{r}\text { Maternal, } \\
\text { Paternal }\end{array}$ & $2.12 \times 10^{-3}$ & Tolerated & Deleterious & Pathogenic & $\begin{array}{l}\text { Disease } \\
\text { causing }\end{array}$ & rs149533049 \\
\hline \multirow{2}{*}{ B3GALTL } & NM_194318:c.660+1G>A & - & \multirow{2}{*}{$\begin{array}{l}\text { Compound } \\
\text { Heterozygous }\end{array}$} & Paternal & $1.09 \times 10^{-3}$ & - & - & $\begin{array}{l}\text { Pathogenic } \\
\text { (high conf.) }\end{array}$ & $\begin{array}{l}\text { Disease } \\
\text { causing }\end{array}$ & rs80338851 \\
\hline & NM_194318:c.755delC & p.Thr252fs*13 & & Maternal & - & - & - & - & $\begin{array}{l}\text { Disease } \\
\text { causing }\end{array}$ & - \\
\hline \multirow{2}{*}{$R Y R 3$} & NM_001036:c.9355G>A & p.Glu3119Lys & \multirow{2}{*}{$\begin{array}{l}\text { Compound } \\
\text { Heterozygous }\end{array}$} & Maternal & $3.06 \times 10^{-3}$ & Tolerated & Deleterious & Pathogenic & $\begin{array}{l}\text { Disease } \\
\text { causing }\end{array}$ & rs200830195 \\
\hline & NM_001036:c.14110G>A & p.Glu4704Lys & & Paternal & $1.08 \times 10^{-2}$ & Tolerated & Neutral & Pathogenic & $\begin{array}{l}\text { Disease } \\
\text { causing }\end{array}$ & rs182257230 \\
\hline \multirow{2}{*}{ KIR2DL3 } & NM_015868:c.44T>G & p.Leu15Trp & Heterozygous & De novo & $1.07 \times 10^{-3}$ & Damaging & Deleterious & Benign & Polymorphism & rs149183658 \\
\hline & NM_015868:c.70+3G>A & - & Heterozygous & De novo & $1.34 \times 10^{-5}$ & - & - & Pathogenic & - & rs776793416 \\
\hline \multirow{2}{*}{ CELSR1 } & NM_014246:c.8282C>T & p.Ser2761Leu & \multirow{2}{*}{$\begin{array}{l}\text { Compound } \\
\text { Heterozygous }\end{array}$} & Maternal & $3.24 \times 10^{-3}$ & Damaging & Deleterious & Pathogenic & $\begin{array}{l}\text { Disease } \\
\text { causing }\end{array}$ & rs144039991 \\
\hline & NM_014246:c.7313G>A & p.Arg2438Gln & & Paternal & $6.34 \times 10-^{4}$ & Tolerated & Neutral & Benign & Polymorphism & rs199688538 \\
\hline
\end{tabular}


Table 2. Results from variant mining with AMELIE and wANNOVAR software. We have showed the top five genes from each software. Variants were sorted according to AMELIE best score.

\begin{tabular}{ccccc}
\hline Gene & Best AMELIE Score & AMELIE Rank & Phenolyzer Score & Phenolyzer Rank \\
\hline B3GLCT & 99.3 & 1 & 0.780 & 1 \\
\hline CELSR1 & 94.2 & 2 & 0.001 & 22 \\
\hline$L R P 8$ & 87.8 & 3 & 0.029 & 4 \\
\hline$L C A T$ & 74.0 & 4 & 0.025 & 8 \\
\hline SHANK3 & 48.3 & 5 & 0.026 & 7 \\
\hline LAMA5 & - & - & 0.041 & 2 \\
\hline SP100 & 0 & 11 & 0.037 & 3 \\
\hline$L A M A 4$ & - & - & 0.028 & 5 \\
\hline
\end{tabular}

\subsection{Sanger Sequencing Results}

For final confirmation of the potentially causative variant in B3GLCT, we performed Sanger sequencing of exons 8 and 9 in the patient and both parents. Both mutations were present in the patient and the inheritance pattern was confirmed. Sanger sequencing electropherograms are presented in Figure 3.

\section{A}

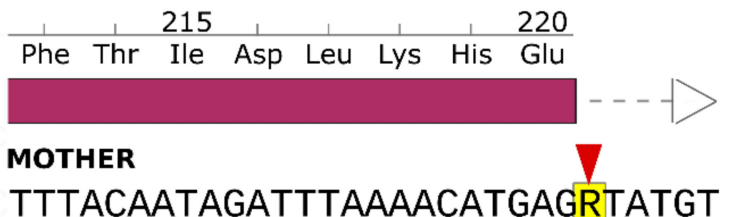

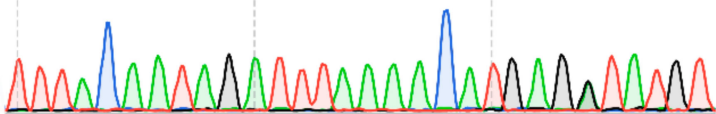

FATHER

TTTACAATAGATTTAAAACATGAGGTATGT

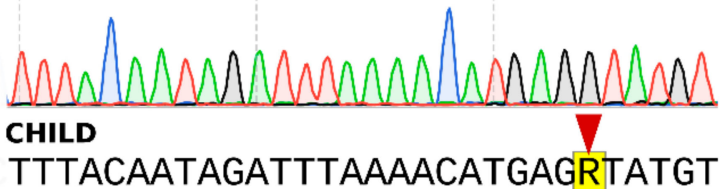

B

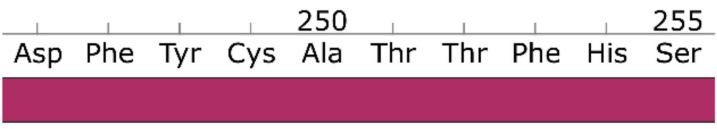

MOTHER

GACTTCTACTGTGCTACCACATTCCATTCT
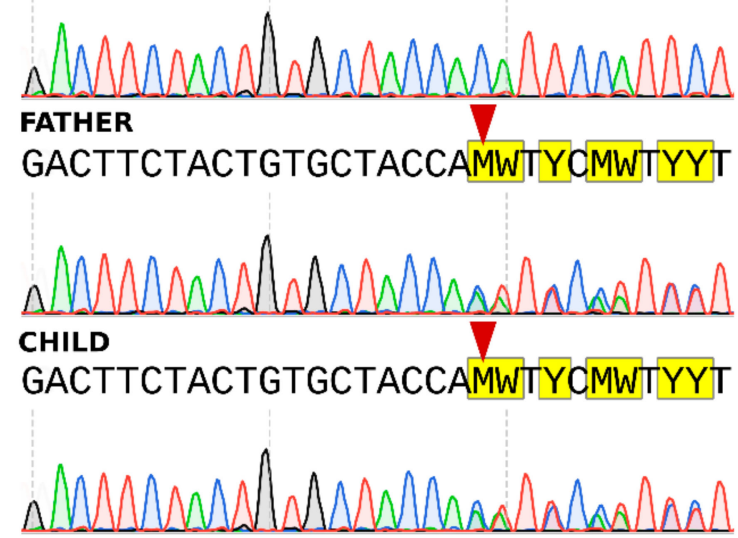

Figure 3. Sanger sequencing electropherograms of B3GLCT exon 8 (A) and exon 9 (B). Points of mutations are indicated with red arrows (violet bar indicates exonic region, white arrow intronic region).

\section{Discussion}

As reported, $6 \%-8 \%$ of children are born with developmental disorders, with many of them caused by genetic changes in a single gene [16]. Despite substantial progress in the NGS data analysis field, finding the causal variant is still a time-consuming process, with the diagnostic yield falling between $25 \%$ and $30 \%$ for exome sequencing data [17-19], especially due to difficulties in the interpretation of the functions of rare variants. In the current study, we used AMELIE and wANNOVAR on NGS data to prioritize genetic variants based on the putative contribution to the clinical phenotype of a pediatric patient suffering from anterior chamber disorder involving corneal opacity, persistent 
pupillary membrane, thinning of the posterior cornea and lenticulo-corneal adhesions with secondary glaucoma and accompanying cleft lip and palate.

Independent of the sequencing scale, NGS creates considerable data burden Thus, careful variant filtering and prioritization, according to the information available from sequencing databases, in compliance with ACMG standards are required. Additionally, differences in NGS data analysis between laboratories could generate discrepancies in the diagnosis, thus filtering schemes should be clearly reported. Moreover, clinical interpretation of sequencing data requires matching information on patients' genotypes and phenotypes and is usually complemented by the integration of information from databases such as OMIM or ClinVar, which are manually constructed and curated. The search for causal variants should be supported by published evidence if it exists, and this requires manual querying of relevant literature databases. This step is the most time-consuming in data processing and has a major impact on timely diagnosis and minimizing false negative and false positive findings. Web-based literature search engines are employed to assist manual curation of newly published information. AMELIE is a method for ranking candidate causal genes related to the phenotype, extracted from primary literature using supervised machine learning methods. The inclusion of HPO-encoded phenotype information facilitates communication with clinical specialists. In our opinion, it combines the convenience of other HPO-based variant ranking tools such as Exomiser [20] with enrichment of the latest literatures. WANNOVAR is a web server which provides functional annotation of genetic variants, reporting their conservation levels, calculating their predicted functional importance scores, retrieving allele frequencies in public databases, and implementing a protocol to identify a subset of potentially deleterious variants/genes.

The combination of these two strategies applied to trio sequencing data led us to the identification of compound heterozygous variants in the B3GLCT gene, which has been previously associated with the PPS phenotype, observed in our patient. Using only a VCF file, annotated by GEMINI with several pathogenicity prediction tools (SIFT, Provean, FATHMM-XF, MutationTaster), resulted in conflicting predictions for variants in some cases. Although the list of possible causative variants included two variants of B3GLCT as compound heterozygous variants, it was not fully informative as the annotation missed the prediction for the deletion NM_194318:c.755delC. Both AMELIE and wANNOVAR ranked variants in genes related to HPO-encoded phenotypes. As a result, B3GLCT got the highest score with both tools. Moreover, in contrast to lower ranked genes, the disease annotated to B3GLCT-PPS-matched our patient's phenotype perfectly.

PPS, a subtype of ASD, inherited in a recessive manner, is a very rare disease with unknown incidence or prevalence. As reported in 2016 by Jaak Jaeken et al., the worldwide number of patients diagnosed with PPS due to B3GLCT mutation was 49 [21]. Common features of PPS involve anterior chamber dysgenesis symptoms such as central corneal clouding, cataract, thinning of the posterior surface of the cornea, iris hypoplasia, and iridocorneal adhesions. In addition to ocular defects, PPS is characterized by short stature, dysmorphic facial features, and developmental delay [21]. In terms of classification, PPS belongs to genetically heterogeneous congenital disorders of glycosylation, grouping rare congenital, neurometabolic, and malformation syndromes [22,23]. It is caused by changes in B3GLCT, encoding an O-fucose-specific $\beta$-1,3-glucosyltransferase (beta-1,3-glucosyltransferase), responsible for attachment of glucose to $O$-linked fucose ( $O$-fucose), which is previously added by protein $O$-fucosyltransferase 2 (POFUT2) to thrombospondin type 1 repeats (TSRs), present in many proteins [24]. Localized at the endoplasmic reticulum (ER) [25], beta-1,3-glucosyltransferase modifies only properly folded TSRs and promotes secretion of ER proteins stabilized by glycosylation. Together with POFUT2, B3GLCT provides a noncanonical quality control system of proper protein folding in cells [26]. A GWAS study on more than 17,100 patients, with advanced age-related macular degeneration (AMD) and over 60,000 controls, showed a significant association between AMD and loci in B3GLCT and ADAMTS9 [27].

On that date, there were 22 unique variants of the B3GLCT gene reported in 10 individuals in the LOVD database. Variant c.660+1G $>$ A (rs80338851), located at the donor splice site (5'ss) of exon 8 
observed in our patient, is one of the most common among variants identified in PPS patients [28]. It has a frequency of $0.01 \%$ in the gnomAD database for European (non-Finnish) population and accounts for $69 \%$ of all reported pathogenic alleles [21]. The results of Oberstein et al. showed that c. $660+1 \mathrm{G}>\mathrm{A}$ alters the acceptor site of exon 8, leading to the skipping of this exon and the introduction of a premature termination codon (PTC) at position +10 within exon 9. According to the position rule for PTC, c.660+1G $>$ A results in a nonsense mRNA that elicits nonsense-mediated mRNA decay (NMD) [29]. NMD is the surveillance pathway protecting cells from the action of truncated proteins which could be translated from transcripts bearing a PTC [30]. In mammalian cells, it is the process of degradation of the mRNA, depending on the interaction between translation termination, complex multiprotein assemblies, which takes place when PTC is present at least 50 nucleotides upstream of the last exon junction. mRNA degradation due to NMD is involved in many neurological and developmental disorders [31].

The second of the detected variants, c.755delC (p.Thr252fs), was not present in any publicly available database and is the first one reported in exon 9. Pathogenicity prediction algorithms assigned this variant as disrupting. Similar to c.660+1G>A, c.755delC introduces a PTC within exon 9, 26 nucleotides from the splice site, which also should result in NMD.

Our study demonstrates the important role and possible diagnostic utility of NGS combined with AMELIE and wANNOVAR.

Such an approach, on the one hand, brings data on genes and variants with well-described roles in disease development based on curated, authoritative databases such as OMIM (wANNOVAR) and, on the other hand, updates the analysis with current information from published manuscripts. Such a procedure helps reduce the number of false positive findings not related to a phenotype which are over-abundant in the VCF file annotated with pathogenicity tools.

\section{Methods}

\subsection{Clinical Assessment}

The patient was recruited from the Clinic of Neonatology at the Jagiellonian University Medical College, Krakow, Poland and the Ophthalmology Clinic and Department of Ophthalmology, Medical University of Silesia in Katowice, Poland. Written informed consent was obtained from the study participants and informed parental consent was obtained on behalf of the child. Developmental and dysmorphology assessments were conducted by a clinical geneticist. This research adhered to the tenets of the Declaration of Helsinki and was performed upon approval of the protocol by the Jagiellonian University Ethics Committee No. 122.6120.12.2015 (29 January 2015).

\subsection{Ophthalmic Evaluation}

The patient was admitted to the outpatient clinic of the Department of Ophthalmology at the Silesian Medical University in Katowice at the age of seven weeks and then, subsequently, at four and six months.

\subsection{DNA Extraction, Library Preparation, and NGS}

DNA was extracted from the peripheral blood of the affected child and both his healthy parents with the Maxwell 16 Blood DNA purification kit (Promega, Madison, WI, USA) on the Maxwell 16 device (Promega). Sequencing libraries were prepared with the TruSight one sequencing panel kit (Illumina, San Diego, CA, USA) according to the manufacturer's protocol. In brief, $50 \mathrm{ng}$ of DNA was fragmented and adaptor-tagged in an enzymatic reaction. Genomic libraries were enriched for the coding regions of the 4813 genes by two cycles of hybridization with biotinylated probes, followed by capture on streptavidin beads; $12.5 \mathrm{pM}$ libraries were sequenced on the MiSeq sequencer (Illumina) using $v 3$ chemistry reagents $(2 \times 150$ bp reads). 


\subsection{Data Analysis}

Raw reads were processed with the Illumina software, generating base calls and corresponding base-call quality scores. These data were then processed through our custom pipeline that uses open-source programs (Figure 4). Briefly, generated fastq files were fed to the FastQC software (version 0.11.5) to provide quality control checks on sequenced data (Andrews et al., 2010). Reads were aligned to the human reference genome GRCh37 (hg19) using the BWA-MEM algorithm from the Burrows Wheeler Aligner (BWA, version 0.7.5) [32]. Unmapped, low mapping quality score and duplicated reads were filtered out with SAMtools (version 0.1.19) [33]. GATK (version 3.7) base quality recalibration was applied across all samples simultaneously using variant quality score recalibration (VQSR) according to the GATK best practices recommendations [34,35]. Filtered variants were concatenated into one record (VCF file) and then, using GEnome MINIng (GEMINI, version 0.18.3) [36], the discovered variants were annotated with SnpEff (version 4.2) [37], and loaded into the SQLite database for parents-child trio analysis. Using the function for trio analysis implemented in the GEMINI software, we identified three groups of variants in the proband patient: (1) De novo, (2) autosomal recessive, and (3) compound heterozygotes. All variants were concatenated into one VCF file and used to predict in silico the effects of amino acid substitutions and indels using SIFT [38], Provean [39], FATHMM_XF [40], and MutationTaster [41]. Simultaneously, the generated VCF file was uploaded into two web tools, Automatic Mendelian Literature Evaluation (AMELIE) [12] and wANNOVAR [15], which connect the ANNOVAR [13] annotation pipeline with the Phenolyzer [42] gene prioritization pipeline. Both softwares were used with standard settings and patient phenotype identifiers as HPO ID. Finally, predicted causative variants were classified according to ACMG2015 guidelines, to verify potential pathogenicity using Varsome [43] online tools.

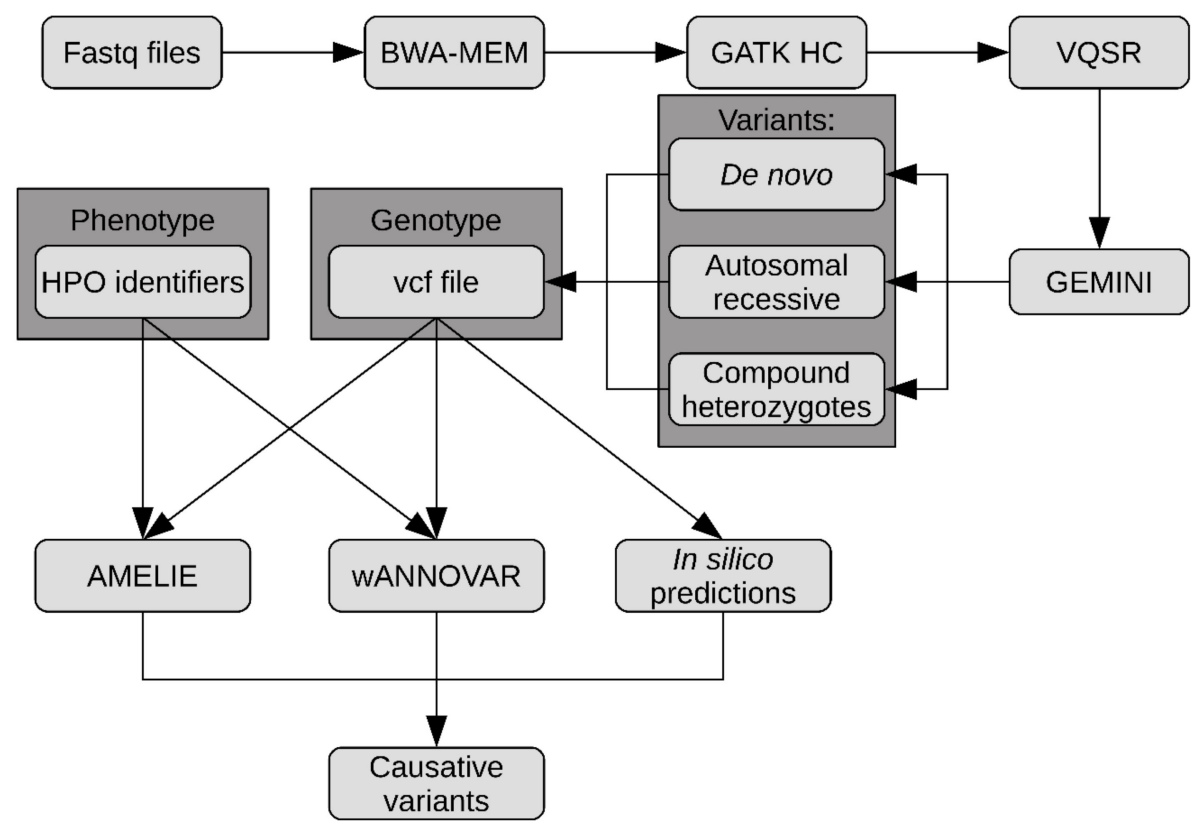

Figure 4. Flow chart describing the steps in the data analysis pipeline.

\subsection{Sanger Sequencing}

For final confirmation of the most plausible causal variants, exons 8 and 9 of B3GLCT (RefSeq NM_194318) were analyzed by Sanger sequencing on the ABI3500 sequencer (Applied Biosystems, ThermoFisher Scientific, Waltham, MA, USA). The obtained sequences were aligned to the reference NC_000013 with the SeqScape software (LifeTechnologies, ThermoFisher Scientific). After identification of an exon-shortening event, the overlapping sequence resulting from the heterozygous deletion was analyzed manually using SnapGene (GSL Biotech, Chicago, IL, USA). 
Supplementary Materials: The following are available online at http://www.mdpi.com/1422-0067/20/23/6006/s1. Supplement 1. VCF file containing variants after trio analysis with GEMINI software. Supplement 2. Gene-term-disease network generated by Phenolyzer, integrated with wANNOVAR web server pipeline.

Author Contributions: J.T.-Ż.; conceptualization, methodology, writing_original draft preparation; P.K.; software, data curation, formal analysis, writing—original draft preparation; M.R.-K.; resources, writing—review and editing; K.L.; resources, writing - review and editing; J.M.; methodology, investigation; A.S.; validation, writing-review and editing; E.F.; resources, writing—review and editing; D.P.; resources, funding acquisition, writing-review and editing; P.P.W.; conceptualization, supervision, funding acquisition, writing-review and editing.

Funding: This research was supported by the statutory funds of the Jagiellonian University Medical College K/ZDS/004416.

Conflicts of Interest: The authors declare no conflict of interest.

\section{References}

1. Richards, S.; Aziz, N.; Bale, S.; Bick, D.; Das, S.; Gastier-Foster, J.; Grody, W.W.; Hegde, M.; Lyon, E.; Spector, E.; et al. Standards and Guidelines for the Interpretation of Sequence Variants: A Joint Consensus Recommendation of the American College of Medical Genetics and Genomics and the Association for Molecular Pathology. Genet. Med. Off. J. Am. Coll. Med. Genet. 2015, 17, 405-424. [CrossRef] [PubMed]

2. Ito, Y.A.; Walter, M.A. Genomics and anterior segment dysgenesis: A review. Clin. Exp. Ophthalmol. 2014, 42, 13-24. [CrossRef] [PubMed]

3. Jun, A.S.; Broman, K.W.; Do, D.V.; Akpek, E.K.; Stark, W.J.; Gottsch, J.D. Endothelial dystrophy, iris hypoplasia, congenital cataract, and stromal thinning (edict) syndrome maps to chromosome 15q22.1-q25.3. Am. J. Ophthalmol. 2002, 134, 172-176.s. [CrossRef]

4. Choi, A.; Lao, R.; Ling-Fung Tang, P.; Wan, E.; Mayer, W.; Bardakjian, T.; Shaw, G.M.; Kwok, P.; Schneider, A.; Slavotinek, A. Novel mutations in PXDN cause microphthalmia and anterior segment dysgenesis. Eur. J. Hum. Genet. 2015, 23, 337-341. [CrossRef] [PubMed]

5. Anand, D.; Agrawal, S.A.; Slavotinek, A.; Lachke, S.A. Mutation update of transcription factor genes FOXE3, HSF4, MAF, and PITX3 causing cataracts and other developmental ocular defects. Hum. Mutat. 2018, 39, 471-494. [CrossRef]

6. Gould, D.B.; Smith, R.S.; John, S.W.M. Anterior segment development relevant to glaucoma. Int. J. Dev. Biol. 2004, 48, 1015-1029. [CrossRef]

7. Micheal, S.; Siddiqui, S.N.; Zafar, S.N.; Villanueva-Mendoza, C.; Cortés-González, V.; Khan, M.I.; den Hollander, A.I. A Novel Homozygous Mutation in FOXC1 Causes Axenfeld Rieger Syndrome with Congenital Glaucoma. PLoS ONE 2016, 11, e0160016. [CrossRef]

8. Weh, E.; Reis, L.M.; Happ, H.C.; Levin, A.V.; Wheeler, P.G.; David, K.L.; Carney, E.; Angle, B.; Hauser, N.; Semina, E.V. Whole exome sequence analysis of Peters anomaly. Hum. Genet. 2014,133,1497-1511. [CrossRef]

9. Meuwissen, M.E.C.; Halley, D.J.J.; Smit, L.S.; Lequin, M.H.; Cobben, J.M.; de Coo, R.; van Harssel, J.; Sallevelt, S.; Woldringh, G.; van der Knaap, M.S.; et al. The expanding phenotype of COL4A1 and COL4A2 mutations: Clinical data on 13 newly identified families and a review of the literature. Genet. Med. Off. J. Am. Coll. Med. Genet. 2015, 17, 843-853. [CrossRef]

10. Favor, J.; Gloeckner, C.J.; Janik, D.; Klempt, M.; Neuhäuser-Klaus, A.; Pretsch, W.; Schmahl, W.; Quintanilla-Fend, L. Type IV Procollagen Missense Mutations Associated with Defects of the Eye, Vascular Stability, the Brain, Kidney Function and Embryonic or Postnatal Viability in the Mouse, Mus musculus: An Extension of the Col4a1 Allelic Series and the Identification of the First Two Col4a2 Mutant Alleles. Genetics 2007, 175, 725-736.

11. Reis, L.M.; Semina, E.V. Genetics of anterior segment dysgenesis disorders. Curr. Opin. Ophthalmol. 2011, 22, 314-324. [CrossRef] [PubMed]

12. Birgmeier, J.; Haeussler, M.; Deisseroth, C.A.; Jagadeesh, K.A.; Ratner, A.J.; Guturu, H.; Wenger, A.M.; Stenson, P.D.; Cooper, D.N.; Re, C.; et al. AMELIE accelerates Mendelian patient diagnosis directly from the primary literature. bioRxiv 2017, 171322.

13. Wang, K.; Li, M.; Hakonarson, H. ANNOVAR: Functional annotation of genetic variants from high-throughput sequencing data. Nucleic Acids Res. 2010, 38, e164. [CrossRef] [PubMed]

14. Chang, X.; Wang, K. wANNOVAR: Annotating genetic variants for personal genomes via the web. J. Med. Genet. 2012, 49, 433-436. [CrossRef] 
15. Yang, H.; Wang, K. Genomic variant annotation and prioritization with ANNOVAR and wANNOVAR. Nat. Protoc. 2015, 10, 1556-1566. [CrossRef] [PubMed]

16. Eisenstein, M. The clinical code-breakers. Nature 2018, 562, 291. [CrossRef] [PubMed]

17. Yang, Y.; Muzny, D.M.; Reid, J.G.; Bainbridge, M.N.; Willis, A.; Ward, P.A.; Braxton, A.; Beuten, J.; Xia, F.; Niu, Z.; et al. Clinical Whole-Exome Sequencing for the Diagnosis of Mendelian Disorders. N. Engl. J. Med. 2013, 369, 1502-1511. [CrossRef]

18. Lee, H.; Deignan, J.L.; Dorrani, N.; Strom, S.P.; Kantarci, S.; Quintero-Rivera, F.; Das, K.; Toy, T.; Harry, B.; Yourshaw, M.; et al. Clinical Exome Sequencing for Genetic Identification of Rare Mendelian Disorders. JAMA 2014, 312, 1880-1887. [CrossRef]

19. Farwell, K.D.; Shahmirzadi, L.; El-Khechen, D.; Powis, Z.; Chao, E.C.; Davis, B.T.; Baxter, R.M.; Zeng, W.; Mroske, C.; Parra, M.C.; et al. Enhanced utility of family-centered diagnostic exome sequencing with inheritance model-based analysis: Results from 500 unselected families with undiagnosed genetic conditions. Genet. Med. 2015, 17, 578-586. [CrossRef]

20. Smedley, D.; Jacobsen, J.O.B.; Jäger, M.; Köhler, S.; Holtgrewe, M.; Schubach, M.; Siragusa, E.; Zemojtel, T.; Buske, O.J.; Washington, N.L.; et al. Next-generation diagnostics and disease-gene discovery with the Exomiser. Nat. Protoc. 2015, 10, 2004-2015. [CrossRef]

21. Jaeken, J.; Lefeber, D.J.; Matthijs, G. Clinical utility gene card for: Peters plus syndrome. Eur. J. Hum. Genet. EJHG 2016, 24, 1232. [CrossRef] [PubMed]

22. Gilfix, B.M. Congenital disorders of glycosylation and the challenge of rare diseases. Hum. Mutat. 2019. [CrossRef] [PubMed]

23. Taylor, R.L.; Arno, G.; Poulter, J.A.; Khan, K.N.; Morarji, J.; Hull, S.; Pontikos, N.; Rueda Martin, A.; Smith, K.R.; Ali, M.; et al. Association of Steroid $5 \alpha$-Reductase Type 3 Congenital Disorder of Glycosylation with Early-Onset Retinal Dystrophy. JAMA Ophthalmol. 2017, 135, 339-347. [CrossRef] [PubMed]

24. Heinonen, T.Y.K.; Mäki, M. Peters'-plus syndrome is a congenital disorder of glycosylation caused by a defect in the $\beta 1,3$-glucosyltransferase that modifies thrombospondin type 1 repeats. Ann. Med. 2009, 41, 2-10. [CrossRef] [PubMed]

25. Sato, T.; Sato, M.; Kiyohara, K.; Sogabe, M.; Shikanai, T.; Kikuchi, N.; Togayachi, A.; Ishida, H.; Ito, H.; Kameyama, A.; et al. Molecular cloning and characterization of a novel human $\beta 1,3$-glucosyltransferase, which is localized at the endoplasmic reticulum and glucosylates O-linked fucosylglycan on thrombospondin type 1 repeat domain. Glycobiology 2006, 16, 1194-1206. [CrossRef]

26. Vasudevan, D.; Takeuchi, H.; Johar, S.S.; Majerus, E.; Haltiwanger, R.S. Peters plus syndrome mutations disrupt a noncanonical ER quality-control mechanism. Curr. Biol. CB 2015, 25, 286-295. [CrossRef]

27. Fritsche, L.G.; Chen, W.; Schu, M.; Yaspan, B.L.; Yu, Y.; Thorleifsson, G.; Zack, D.J.; Arakawa, S.; Cipriani, V.; Ripke, S.; et al. Seven new loci associated with age-related macular degeneration. Nat. Genet. 2013, 45, 433-439.

28. Lesnik Oberstein, S.A.J.; Kriek, M.; White, S.J.; Kalf, M.E.; Szuhai, K.; den Dunnen, J.T.; Breuning, M.H.; Hennekam, R.C.M. Peters Plus syndrome is caused by mutations in B3GALTL, a putative glycosyltransferase. Am. J. Hum. Genet. 2006, 79, 562-566. [CrossRef]

29. Nagy, E.; Maquat, L.E. A rule for termination-codon position within intron-containing genes: When nonsense affects RNA abundance. Trends Biochem. Sci. 1998, 23, 198-199. [CrossRef]

30. Lindeboom, R.G.H.; Supek, F.; Lehner, B. The rules and impact of nonsense-mediated mRNA decay in human cancers. Nat. Genet. 2016, 48, 1112-1118. [CrossRef]

31. Khajavi, M.; Inoue, K.; Lupski, J.R. Nonsense-mediated mRNA decay modulates clinical outcome of genetic disease. Eur. J. Hum. Genet. EJHG 2006, 14, 1074-1081. [CrossRef] [PubMed]

32. Li, H.; Durbin, R. Fast and accurate long-read alignment with Burrows-Wheeler transform. Bioinforma. Oxf. Engl. 2010, 26, 589-595. [CrossRef] [PubMed]

33. Li, H.; Handsaker, B.; Wysoker, A.; Fennell, T.; Ruan, J.; Homer, N.; Marth, G.; Abecasis, G.; Durbin, R. 1000 Genome Project Data Processing Subgroup the Sequence Alignment/Map format and SAMtools. Bioinforma. Oxf. Engl. 2009, 25, 2078-2079. [CrossRef] [PubMed]

34. DePristo, M.A.; Banks, E.; Poplin, R.; Garimella, K.V.; Maguire, J.R.; Hartl, C.; Philippakis, A.A.; del Angel, G.; Rivas, M.A.; Hanna, M.; et al. A framework for variation discovery and genotyping using next-generation DNA sequencing data. Nat. Genet. 2011, 43, 491-498. [CrossRef] [PubMed] 
35. Van der Auwera, G.A.; Carneiro, M.O.; Hartl, C.; Poplin, R.; Del Angel, G.; Levy-Moonshine, A.; Jordan, T.; Shakir, K.; Roazen, D.; Thibault, J.; et al. From FastQ data to high confidence variant calls: The Genome Analysis Toolkit best practices pipeline. Curr. Protoc. Bioinform. 2013, 43, 11.10.1-11.10.33.

36. Paila, U.; Chapman, B.A.; Kirchner, R.; Quinlan, A.R. GEMINI: Integrative Exploration of Genetic Variation and Genome Annotations. PLOS Comput. Biol. 2013, 9, e1003153. [CrossRef]

37. Cingolani, P.; Platts, A.; Wang, L.L.; Coon, M.; Nguyen, T.; Wang, L.; Land, S.J.; Lu, X.; Ruden, D.M. A program for annotating and predicting the effects of single nucleotide polymorphisms, SnpEff: SNPs in the genome of Drosophila melanogaster strain w1118; iso-2; iso-3. Fly (Austin) 2012, 6, 80-92. [CrossRef]

38. Vaser, R.; Adusumalli, S.; Leng, S.N.; Sikic, M.; Ng, P.C. SIFT missense predictions for genomes. Nat. Protoc. 2016, 11, 1-9. [CrossRef]

39. Choi, Y.; Sims, G.E.; Murphy, S.; Miller, J.R.; Chan, A.P. Predicting the functional effect of amino acid substitutions and indels. PLoS ONE 2012, 7, e46688. [CrossRef]

40. Rogers, M.F.; Shihab, H.A.; Mort, M.; Cooper, D.N.; Gaunt, T.R.; Campbell, C. FATHMM-XF: Accurate prediction of pathogenic point mutations via extended features. Bioinformatics 2018, 34, 511-513. [CrossRef]

41. Schwarz, J.M.; Cooper, D.N.; Schuelke, M.; Seelow, D. MutationTaster2: Mutation prediction for the deep-sequencing age. Nat. Methods 2014, 11,361-362. [CrossRef] [PubMed]

42. Yang, H.; Robinson, P.N.; Wang, K. Phenolyzer: Phenotype-based prioritization of candidate genes for human diseases. Nat. Methods 2015, 12, 841-843. [CrossRef] [PubMed]

43. Kopanos, C.; Tsiolkas, V.; Kouris, A.; Chapple, C.E.; Albarca Aguilera, M.; Meyer, R.; Massouras, A. VarSome: The human genomic variant search engine. Bioinformatics 2019, 35, 1978-1980. [CrossRef] [PubMed]

(C) 2019 by the authors. Licensee MDPI, Basel, Switzerland. This article is an open access article distributed under the terms and conditions of the Creative Commons Attribution (CC BY) license (http://creativecommons.org/licenses/by/4.0/). 